\title{
Effets de Dix (10) Variétés de Sésame (Sesamum indicum (L.)) en Association avec Le Niébé (Vigna unguiculata (L.) Walp) dans la Lutte Contre Striga Gesnerioïdes (Willd.) Vatke. au Niger
}

\author{
Amadou Mounkaila Hamissou \\ Amoukou Adamou Ibrahim \\ Zangui Hamissou
}

Département de Productions Végétales, Faculté d’Agronomie, Université

Abdou Moumouni de Niamey, Niamey, Niger

\section{Boureima Seyni}

Département de Productions Végétales Faculté d’Agronomie et des Sciences de l’Environnement, Université Dan Dicko Dankoulodo de Maradi, Maradi, Niger.

\section{Naino Jika Abdel Kader}

Alliance of Bioversity International and International Center for Tropical Agriculture (CIAT)

Headquarters, via dei Tre Denari, Maccarese, Rome, Italy

\section{Doi:10.19044/esj.2021.v17n37p15}

Submitted: 23 August 2021

Accepted: 12 October 2021

Published: 31 October 2021
Copyright 2021 Author(s)

Under Creative Commons BY-NC-ND 4.0 OPEN ACCESS

Cite As:

Amadou Mounkaila H., Amoukou Adamou I., Zangui H., Boureima S. \& Naino Jika A.K. (2021). Effets de Dix (10) Variétés de Sésame (Sesamum indicum (L.)) en Association avec Le Niébé (Vigna unguiculata (L.) Walp) dans la Lutte Contre Striga Gesnerioïdes (Willd.) Vatke. au Niger. European Scientific Journal, ESJ, 17(37), 15.

https://doi.org/10.19044/esj.2021.v17n37p15

\section{Résumé}

Striga gesnerioïdes est une plante parasite qui cause des dégâts importants sur le niébé et les méthodes de lutte testées restent insatisfaisantes. L'étude vise à mettre au point, une référence de technique d'association sésame-niébé pour contribuer à lutter efficacement contre Striga gesnerioïdes. À cet effet, un dispositif en Split-Plot est utilisé sur un terrain naturellement infesté par Striga gesnerioïdes. Trois comptages à des périodes différentes (42, 56 et 70 jours après semis) du nombre de plants de Striga gesnerioïdes émergés par poquet de niébé ont été réalisés. Ainsi, en association dans le 
même poquet (sésame + niébé), les variétés de sésame EF 146, HC 110, ICN 130, HB 168, Vgr 156 et EF 147 sont efficaces pour atténuer l'émergence de striga. En alternance de poquets (niébé et sésame), les variétés de sésame EF 146, HC 110, ICN 137, EF 147 et 38-1-7 sont efficaces contre striga. Et les variétés de sésame EF 146, Vgr 156, HC 108 et 38-1-7 ont montré leurs efficacités en alternance de lignes avec le niébé. EF 146 est la seule variété de sésame efficace dans toutes les trois mesures de protections. Ces résultats pourraient être vulgarisés au niveau des producteurs de niébé pour booster sa production au niveau national, voir mondial.

Mots clés: Striga gesnerioïdes, Sesamum indicum, Vigna unguiculata, Plante Piège, Niger

\title{
Effects of Ten (10) Varieties of Sesame (Sesamum Indicum (L.)) in Association With Cowpea (Vigna Unguiculata (L.) Walp) in The Control of Striga Gesnerioïdes (Willd.) Vatke in Niamey, in Niger
}

\section{Amadou Mounkaila Hamissou Amoukou Adamou Ibrahim Zangui Hamissou}

Département de Productions Végétales, Faculté d’Agronomie, Université Abdou Moumouni de Niamey, Niamey, Niger

\section{Boureima Seyni}

Département de Productions Végétales Faculté d'Agronomie et des Sciences de l’Environnement, Université Dan Dicko Dankoulodo de Maradi, Maradi, Niger.

\section{Naino Jika Abdel Kader}

Alliance of Bioversity International and International Center for Tropical Agriculture (CIAT)

Headquarters, via dei Tre Denari, Maccarese, Rome, Italy

\begin{abstract}
Striga gesnerioïdes is a parasitic plant that causes significant damage to cowpea and the control methods tested remain unsatisfactory. The study aims to develop a technical reference of sesame-cowpea association to contribute to the effective control of Striga gesnerioïdes. For this purpose, a Split-Plot device is used on land naturally infested by Striga gesnerioïdes. Three counts at different periods (42, 56 and 70 days) of the number of emerged Striga gesnerioïdes plants per cowpea were made. Thus, in combination in the same plot (sesame + cowpea), the sesame varieties EF 146,
\end{abstract}


HC 110, ICN 130, HB 168, Vgr 156 and EF 147 are effective in mitigating Striga emergence. In alternating rows (cowpea and sesame), the sesame varieties EF 146, HC 110, ICN 137, EF 147 and 38-1-7 were effective against striga. And the sesame varieties EF 146, Vgr 156, HC 108 and 38-1-7 showed their efficacy in alternating rows with cowpea. EF 146 is the only sesame variety that is effective in all three protective measures. These results could be disseminated to cowpea producers to boost its production at the national and even global level.

Keywords: Striga gesnerioides, Sesamum indicum, Vigna unguiculata, Trap Plant, Niger

\section{Introduction}

Tout comme le niébé, le sésame occupe sur le plan mondial une place importante et joue des fonctions multiples. C'est le cas de ses grains qui sont utilisés dans la pâtisserie, pour extraire de l'huile de haute qualité et aussi dans la fabrication des produits cosmétiques. L'huile de sésame possède des propriétés thérapeutiques (Boureima et al., 2012; Okandza et al., 2017; Pathak et al., 2017).

Outre ces fonctions, le sésame joue un rôle important sur le plan agroécologique (Hamissou et al., 2020). En effet, un savoir endogène des producteurs de niébé, confère au sésame le rôle de plante piège contre Striga gesnerioïdes. Cette plante parasite redoutable occasionne des pertes de rendements en grains de niébé au Sahel d'environ $75 \%$ de la production au niveau national et à $100 \%$ au niveau d'un champ en Afrique de l'Ouest (Kgosi et al., 2012 ; Spallek et al., 2013). À cet effet, plusieurs méthodes de lutte (physique, mécanique, chimique et culturale) sont expérimentées sans succès (Olivier, 2008) avec des insuffisances d'ordre social, économique et environnemental (Zia et Khan, 2002 ; Olupot et al., 2003). La lutte biologique contre les plantes parasites est inscrite dans le concept de la gestion intégrée des mauvaises herbes en malherbologie qui fait appel généralement, à l'utilisation des microorganismes (bactérie, champignon, virus) et en particulier des organismes (plante piège) (Waters et al., 2017; Samejima et al., 2018 ; Kondi et al., 2018). En milieu paysan, le sésame est utilisé comme plantes pièges de Striga gesnerioïdes à travers des techniques d'association avec les plantes hôtes comme le niébé. Ainsi, cette étude porte sur « Effets de dix (10) variétés de sésame (Sesamum indicum (L.)) en association avec le niébé (Vigna unguiculata (L.) Walp) dans la lutte contre Striga gesnerioïdes (Willd.) Vatke au Niger ». Le but de cette étude est de mettre en place une référence en technique d'association niébé-sésame pour lutter efficacement contre Striga gesnerioïdes. 


\section{Matériel et Méthodes \\ Site expérimental}

L’essai a été conduit sur le site expérimental de la Faculté d’Agronomie de l'Université Abdou Moumouni de Niamey entre la latitude Nord $13^{\circ} 29^{\prime} 56^{\prime}$ ', la longitude Est $02^{\circ} 05^{\prime} 30^{\prime \prime}$ et l'altitude 206,35 m pendant la campagne d'hivernage 2019.

\section{Matériel végétal}

Le matériel végétal utilisé est constitué de la variété de niébé IT 90 K372-1-2 et de dix (10) variétés de sésame à savoir : HC108, HC110, ICN137, ICN130, HB168, Vgr 156, Birkan, 38-1-7, EF147 et EF146.

\section{Méthodologie}

L'essai a été conduit sur un site naturellement infesté de Striga gesnerioïdes. Un dispositif expérimental en Split-Plot à deux répétitions a été utilisé sur une superficie de $2354 \mathrm{~m}^{2}$ (53,5 m x $44 \mathrm{~m}$ ), avec une densité de semis de $1 \mathrm{~m} \mathrm{x} 1 \mathrm{~m}$. Il est constitué d'un facteur principal (variétés de sésame) et d'un facteur secondaire (les traitements). Les traitements sont : traitement 1 : association sésame + niébé dans le même poquet ; traitement 2 : alternance d'un poquet de sésame et d'un poquet de niébé ; traitement 3 : alternance d'une ligne de sésame et d'une ligne de niébé ; traitement 4 (témoin) : culture pure de sésame et traitement 5 (témoin) : culture pure niébé. Conduite de la culture : Le semi a été réalisé après une pluie utile de 13,5 mm. Le démariage a été effectué à deux plants / poquet pour chaque espèce (sésame et niébé). Deux semaines après la levée, un seul sarclage est réalisé suivi par l'application de $5 \mathrm{~g}$ par poquet d'engrais NPK (15-15-15) en micro-dose localisée. Un désherbage hebdomadaire manuel et continu a été réalisé en cas de besoins jusqu'à la fin de l'essai.

\section{Observations}

Les plants de Striga gesnerioïdes émergés par poquet de niébé sont comptés à trois périodes du cycle à savoir 42, 56 et 70 jours après semis (JAS).

\section{Analyses statistiques}

Une analyse de variance au seuil de $5 \%$ est faite sur les données du nombre de plants de Striga gesnerioïdes émergés avec le logiciel « Genstat $9^{\text {th }}$ Edition ». Pour les résultats significatifs, un test de comparaison de moyennes est réalisé avec la méthode de Duncan.

\section{Résultats}

L’analyse de variance (Tableau I) réalisé sur le nombre de striga émergés à trois (3) périodes du cycle de la culture (42, 56 et 70 JAS) montre 
que l'infestation du striga n'est pas homogène dans les blocs. Ce qui prouve qu'il y a une hétérogénéité de l'inoculum du parasite sur le terrain d'expérimentation, qui est observable dès le $56^{\text {èm }}$ JAS (observation médiane).

En revanche, l'effet variétal de sésame n'est significatif qu'à 70 JAS (dernière observation), alors que celui des techniques d'associations est

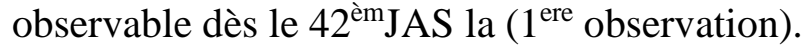

L'interaction variété de sésame et techniques d'associations ne devient significatif que vers la fin du cycle à partir de $70^{\mathrm{èm}} \mathrm{JAS}$ (dernière observation).

Tableau I. Analyse de variance ( $1^{\text {ère }}$; médiane et dernière observations)

\begin{tabular}{|c|c|c|c|c|c|c|c|c|c|}
\hline & \multicolumn{3}{|c|}{$\begin{array}{c}1^{\text {ere }} \text { observation (42 } \\
\text { JAS) }\end{array}$} & \multicolumn{3}{|c|}{$\begin{array}{c}\text { Observation médiane } \\
\text { (56 JAS) }\end{array}$} & \multicolumn{3}{|c|}{$\begin{array}{c}\text { Dernière observation (70 } \\
\text { JAS) }\end{array}$} \\
\hline Origine de variation & $\begin{array}{c}\text { d.d. } \\
\text { l }\end{array}$ & $\bar{F}$ & $\mathrm{Pr}$ & d.d.l & $\mathrm{F}$ & $\operatorname{Pr}$ & d.d.l & $\mathrm{F}$ & $\mathrm{Pr}$ \\
\hline Blocs & 1 & 0,29 & & 1 & 0,02 & & 1 & 00,0 & \\
\hline \multicolumn{10}{|c|}{ Blocs x Sous blocs } \\
\hline Variétés & 9 & 1,30 & 0,350 & 9 & 2,38 & 0,106 & 9 & 3,35 & 0,043 \\
\hline Résiduelle & 9 & 1,03 & & 9 & 2,07 & & 9 & 3,01 & \\
\hline \multicolumn{10}{|c|}{ Blocs x Sous blocs x Parcelles } \\
\hline $\begin{array}{c}\text { Techniques } \\
\text { d'associations }\end{array}$ & 4 & 4,54 & 0,004 & 4 & 20,39 & 0,001 & 4 & 43,98 & 0,001 \\
\hline $\begin{array}{c}\text { Techniques associations } \\
\text { x Variétés }\end{array}$ & 36 & 0,68 & 0,879 & 36 & 1,52 & 0,099 & 36 & 3,81 & 0,001 \\
\hline Résiduelle & 40 & & & 40 & & & 40 & & \\
\hline Total & 99 & & & 99 & & & 99 & & \\
\hline
\end{tabular}

Légende: d.d.l.: degré de liberté ; F: valeur du test Fisher ; Pr.: probabilité observée ; JAS:

Les comparaisons des moyennes des combinaisons techniques d'associations X sésames (Tableau II) montrent que c'est avec les variétés de sésames EF 146, HB 168, HC110, ICN 130 et Vgr 156 que les meilleurs effets d'atténuation de l'infestation de striga sont obtenus et ceux quel que soit le type d'association. D'ailleurs, même la tendance montre que c'est dans le même poquet que l'effet est meilleur. En revanche, les autres variétés de sésame (38-1-7, Birkan, EF 147, HC 108 et ICN 137) ont tendance à stimuler l'émergence du striga et surtout quand elles sont associées dans les mêmes poquets avec le niébé par rapport aux alternances (alternance de poquets et alternance de lignes) de sésame avec le niébé. 
Tableau II. Comparaison des moyennes du nombre de plants de Striga gesneriö̈des émergés par poquet de niébé (70 JAS) et groupes homogènes

\begin{tabular}{|c|c|c|c|c|c|c|c|c|c|c|}
\hline \multicolumn{11}{|c|}{ Techniques d'associations } \\
\hline $\begin{array}{c}\text { Techniques } \\
\text { Associations }\end{array}$ & \multicolumn{2}{|c|}{$\begin{array}{l}\text { Alternance } \\
\text { Lignes }\end{array}$} & \multicolumn{2}{|c|}{ Alternance Poquets } & \multicolumn{2}{|c|}{ Dans Poquets } & \multicolumn{2}{|c|}{ Témoin Niébé } & \multicolumn{2}{|c|}{ Témoin Sésame } \\
\hline Moyennes & \multicolumn{2}{|c|}{$22 \mathrm{~B}$} & \multicolumn{2}{|c|}{$23 \mathrm{~B}$} & \multicolumn{2}{|c|}{$41 \mathrm{C}$} & \multicolumn{2}{|c|}{$56 \mathrm{D}$} & \multicolumn{2}{|c|}{$0 \mathrm{~A}$} \\
\hline \multicolumn{11}{|c|}{ Variétés de sésame } \\
\hline Variétés & $38-1-7$ & $\begin{array}{c}\text { Birka } \\
\text { n }\end{array}$ & EF 146 & EF 147 & HB 168 & HC 108 & HC 110 & ICN 130 & ICN 137 & $\operatorname{Vgr} 156$ \\
\hline Moyennes & $33 \mathrm{AB}$ & $42 \mathrm{~B}$ & $13 \mathrm{~A}$ & $45 \mathrm{~B}$ & $11 \mathrm{~A}$ & $45 \mathrm{~B}$ & $14 \mathrm{~A}$ & $12 \mathrm{~A}$ & $35 \mathrm{AB}$ & $36 \mathrm{AB}$ \\
\hline \multicolumn{11}{|c|}{ Techniques d'association $\mathbf{x}$ Variétés de sésame } \\
\hline & 38-1-7 & $\begin{array}{c}\text { Birka } \\
\text { n }\end{array}$ & EF 146 & EF 147 & HB 168 & HC 108 & HC 110 & ICN 130 & ICN 137 & Vgr 156 \\
\hline $\begin{array}{l}\text { Alternance } \\
\text { Lignes }\end{array}$ & $25 \mathrm{AB}$ & $27 \mathrm{AB}$ & $14 \mathrm{~A}$ & $44 \mathrm{BC}$ & $13 \mathrm{~A}$ & $35 \mathrm{~B}$ & $14 \mathrm{~A}$ & $13 \mathrm{~A}$ & $20 \mathrm{AB}$ & $18 \mathrm{~A}$ \\
\hline $\begin{array}{l}\text { Alternance } \\
\text { Poquets }\end{array}$ & $23 \mathrm{AB}$ & $43 \mathrm{BC}$ & $13 \mathrm{~A}$ & $29 \mathrm{AB}$ & $10 \mathrm{~A}$ & $52 \mathrm{BC}$ & $15 \mathrm{~A}$ & $14 \mathrm{~A}$ & $26 \mathrm{AB}$ & $10 \mathrm{~A}$ \\
\hline Dans Poquets & $28 \mathrm{AB}$ & $99 \mathrm{CD}$ & $5 \mathrm{~A}$ & $45 \mathrm{BC}$ & $6 \mathrm{~A}$ & $82 \mathrm{C}$ & $11 \mathrm{~A}$ & $10 \mathrm{~A}$ & $38 \mathrm{~B}$ & $9 \mathrm{~A}$ \\
\hline Témoin Niébé & $91 \mathrm{C}$ & $40 \mathrm{~B}$ & $32 \mathrm{AB}$ & $107 \mathrm{CD}$ & $29 \mathrm{AB}$ & $58 \mathrm{BC}$ & $29 \mathrm{AB}$ & $26 \mathrm{AB}$ & $93 \mathrm{C}$ & $59 \mathrm{BC}$ \\
\hline Témoin Sésame & $0 \mathrm{~A}$ & $0 \mathrm{~A}$ & $0 \mathrm{~A}$ & $0 \mathrm{~A}$ & $0 \mathrm{~A}$ & $0 \mathrm{~A}$ & $0 \mathrm{~A}$ & $0 \mathrm{~A}$ & $0 \mathrm{~A}$ & $0 \mathrm{~A}$ \\
\hline
\end{tabular}

\section{Discussion}

Striga gesnerioïdes est une plante parasite redoutable des légumineuses. $\mathrm{Au}$ Niger, cette plante parasite infeste les terres arables destinées à la production de niébé. Cette étude a été réalisée sur un terrain avec une infestation naturelle et hétérogène de Striga gesnerioïdes, qui est

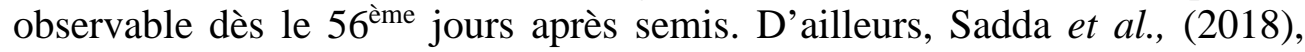
montrent que la répartition de l'infestation naturelle de la plante parasite Striga gesnerioïdes (Willd.) Vatke est localisée et varie d’une manière générale en fonction des zones de production au Niger.

Les variétés de sésame EF 146, HC 110, ICN 130, HB 168 et Vgr 156 qui provoquent un effet variétal positif de plantes pièges contre Striga gesnerioïdes corroborent les travaux de Ouédraogo et al. (2018) qui ont montré l'existence d'une plante piège Polygala rarifolia, contre Striga hermonthica, avec comme effet une réduction efficace de la germination et du développement de la plante parasite. Ces résultats confirment aussi l'effet de plante piège de sésame contre Striga hermonthica (Hamissou et al., 2020).

Dembele (1988) ; Kayentao (2008) ; Siame et al. (1993) ont longtemps préconisé plusieurs plantes pièges en Afrique de l'Ouest, parmi lesquelles le soja, l'arachide, le coton et l'oseille. Les espèces pérennes comme Cassia obtusifolia et Cardiospermum halicacabum ont longtemps aussi été utilisées comme plantes pièges de Striga (Dubé et Olivier, 2001 ; Lado et al., 2018; Leandre et al., 2018).

Il ressort des résultats que l'efficacité de l'utilisation de la combinaison de sésame et de niébé pour le contrôle de Striga gesnerioïdes dépend des 
variétés de sésame et du type de technique d'association utilisée. C'est ainsi que les variétés de sésame EF 146, HC 110, ICN 130, HB 168, Vgr 156 et EF 147 sont efficaces pour atténuer l'émergence de Striga gesneriö̈des surtout en association dans le même poquet avec le niébé. Les variétés de sésame EF 146, HC 110, ICN 137, EF 147 et 38-1-7 sont efficaces contre striga en alternance de poquet avec le niébé. Les variétés de sésame EF 146, Vgr 156, HC 108 et 38-1-7 montrent aussi leur efficacité en alternance de lignes avec le niébé. Selon Hamissou et al. (2020), l'utilisation de l'une de ces trois variétés de sésame (ICN 130, EF 146 et HC 110) en association avec le mil (variété HKP) dans le même poquet réduit de moitié l'émergence de Striga hermonthica. D'ailleurs les études de Lado et al.(2018) et Sundar et al.(2018) montrent que le niébé en association avec des pieds de Parkia biglobosa diminue considérablement l'effet envahissant de Striga gesnerioïdes. Bien que, l'accent ait été longtemps mis sur l'effet des variétés résistantes de niébé contre Striga gesnerioïdes plutôt que l'utilisation des plantes pièges contre Striga gesnerioïdes (Kondi et al., 2018 ; Leandre et al., 2018).

\section{Conclusion}

L'étude montre que les mesures de protections du niébé contre Striga gesnerioïdes, qui utilisent le sésame et le niébé en association (dans le même poquet) ou en alternance (de lignes ou de poquets) peuvent être efficaces. Toutefois, l'efficacité de ces techniques d'association dépend de la variété de sésame utilisée et de l'architecture d'association sésame-niébé utilisée. C'est ainsi que les variétés de sésame EF 146, HC 110, ICN 130, HB 168, Vgr 156 et EF 147 sont efficaces pour atténuer l'émergence de Striga gesnerioïdes dans le cas de l'association dans le même poquet avec le niébé. Les variétés de sésame EF 146, HC 110, ICN 137, EF 147 et 38-1-7 sont efficaces contre striga en alternance de poquet avec le niébé. Et les variétés de sésame EF 146, Vgr 156, HC 108 et 38-1-7 ont montré leur efficacité en alternance de lignes avec le niébé. Cependant, une seule variété de sésame EF 146 a montré son efficacité dans toutes les trois mesures de protections de niébé contre Striga gesnerioïdes. Ces résultats peuvent être mis à la disposition des producteurs de niébé par le biais de la vulgarisation.

\section{Remerciements}

Les auteurs remercient le projet «Amélioration de la productivité et valorisation du sésame (Sesamum indicum (L.)) au Niger » financé par le Programme Productivité Agricole en Afrique de l'Ouest (PPAAO) et la Faculté d’Agronomie de l’Université Abdou Moumouni de Niamey. 


\section{References:}

1. Boureima, S., Diouf, M. and Cissé, N. (2012). Besoin en eau, croissance et rendement du sésame en zone semi-aride. Agronomie Africaine 22 (2): 139-147.

2. Dembele, B. (1988). "Aspects biologiques et agronomiques de deux scrophulariacées parasites tropicales: Striga hermonthica (Del.) Benth. et Striga gesnerioides (Willd.) Vatke." Ecole Normale Supérieure Agronomique de Montpellier, Doctor Thesis 1: 100.

3. Dubé, M.-P. and A. Olivier (2001). "Le Striga gesnerioides et son hôte, le niébé: interaction et méthodes de lutte." Canadian Journal of Botany 79(10): 1225-1240.

4. Hamissou, A. M., Ibrahim, A. A., \& Hamissou, Z. (2020). Effet du sésame (Sesamum indicum L.) sur le développement de Striga hermonthica (Del.) Benth. Journal of Applied Biosciences, 152, 15720-15726.

5. Kayentao, M. (2008). "Gestion de Striga au Mali." progress on farmers training on parasitic weed management: 111.

6. Kgosi, R. L., Zwanenburg, B., Mwakaboko, A. S., \& Murdoch, A. J. (2012). Strigolactone nalogues induce suicidal seed germination of Striga spp. in soil. Weed Research, 52(3), 197-203.

7. Kondi, Y., A. Mawuli, B. Agnassim, P. Yentchabre, T. Koffy. (2018). "Evaluation De Quatre Cultivars De Niebe (Vigna Unguiculata (L.) Walp.) Pour Leur Resistance Au Deficit Hydrique Et A L'adventice Parasite, Striga gesnerioides (Willd.) Vatke $\mathrm{Au}$ Togo." European Scientific Journal, ESJ 14(6).

8. Lado, A., Sani, F. U., Yahaya, S. U., \& Karaye, A. K. (2018). Efficacy of Parkia biglobosa fruit powder on the control of Striga in cowpea cropping systems in the Sudan-Savanna, Nigeria. Heliyon, 4(8), e00733..

9. Leandre, S. P., Francis, K., Richard, A., Joseph, B., Ouedraogo, J. T., Patrick, A., ... \& Roberbs, P. A. (2018). Screening for resistance to Striga gesnerioïdes and estimation of yield loss among Cowpea (Vigna unguiculata (L.) Walp.) progenies in the Upper East Regionof Ghana. African Journal of Agricultural Research, 13(28), 1430-1442.

10. Okandza, Y., Ossoko, J. P., Yoca, J. E., Dzondo, G. M., Tsieri, M. M., Abdenour, Y., \& Toubate, B. (2017). Identification des tocophérols, Stérols, alcools aliphatiques et terpéniques de l'huile de sésame (Sesamum indicum (L.)) de la République du Congo. International Journal of Innovation and Applied Studies, 19(1), 79.

11. Olivier de Sardan, J.-P. (2008). "La rigueur du qualitatif. Les contraintes empiriques de l'interprétation socio-anthropologique." Lectures, Publications reçues. 
12. Olupot, J. R., Osiru, D. S. O., Oryokot, J., \& Gebrekidan, B. (2003). The effectiveness of Celosia argentia (Striga "chaser") to control Striga on Sorghum in Uganda. Crop protection, 22(3), 463-468.

13. Ouédraogo, O., Kaboré, T. D., Noba, D. R., \& Traoré, S. (2018). Polygala rarifolia DC., plante faux hôte du Striga hermonthica (Del.) Benth. Journal of Applied Biosciences, 123, 12346-12353.

14. Pathak, K., Rahman, S. W., Bhagawati, S., \& Gogoi, B. (2017). Sesame (Sesamum indicum L.), an underexploited oil seed crop: Current status, features and importance-A review. Agricultural Reviews, 38(3).

15. Sadda, A. S., Diouf, A., Salifou Jangorzo, N., Issoufou Hassane, B. A., Saidou, A. A., Karim, S., \& Malam-Issa, O. (2018). Modélisation prédictive d'un parasite des variétés de niébé au Niger : cas du Striga gesnerioïdes (Willd.) Vatke. INRA.

16. Samejima, H., Babiker, A. G., \& Sugimoto, Y. (2018). Amélioration de la sécurité alimentaire dans les régions semi-arides du Soudan grâce à la gestion des mauvais parasites des racines. Dans la production agricole dans des conditions stressantes (pp. 159-175). Springer, Singapour.

17. Siame, B. A., Weerasuriya, Y., Wood, K., Ejeta, G., \& Butler, L. G. (1993). Isolation of strigol, a germination stimulant for Striga asiatica, from host plants. Journal of Agricultural and Food Chemistry, 41(9), 1486-1491.

18. Spallek, T., Mutuku, M., \& Shirasu, K. (2013). The genus S triga: a witch profile. Molecular plant pathology, 14(9), 861-869.

19. Sundar, R. D. V., Settu, S., Shankar, S., Segaran, G., \& Sathiavelu, M. (2018). Plantes médicinales potentielles pour traiter la lèpre-A Examen. Research Journal of Pharmacy and Technology, 11(2), 813821.

20. Waters, M. T., Gutjahr, C., Bennett, T., \& Nelson, D. C. (2017). Strigolactone signaling and evolution. Annual review of plant biology, 68, 291-322.

21. Zia, S. and M. A. Khan (2002). "Comparative effect of $\mathrm{NaCl}$ and seawater on seed germination of Limonium stocksii." Pakistan Journal of Botany 34: 345-350.

22. Site: http://www.fao.org/faostat/fr/\#data/QC, visité le 25/12/2018 à 17H03 haire locale (Niamey-Niger, dernière mise à jours, 20/03/2021. 